\title{
Was leisten unsere Schüler am Ende der Grundschulzeit in Mathematik? von Gerd Walther
}

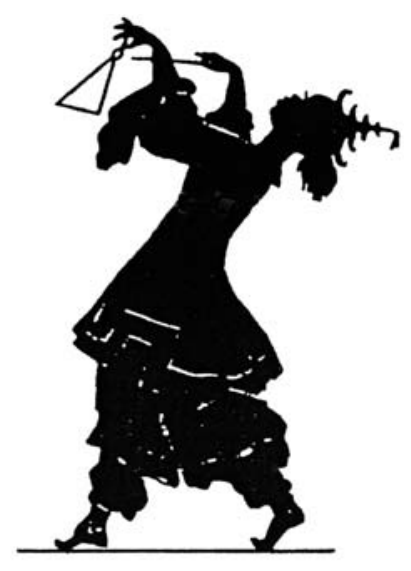

DER GEOMETRIE UNTER RICHTFANGT AN!

Kuni

\begin{abstract}
Als ich gebeten wurde, einen Beitrag für die Mitteilungen zu schreiben, habe ich mich natürlich gefragt, was Leser dieser Zeitschrift gerade an dieser Frage interessieren könnte. Sofern eigene oder einem nahe stehende Kinder die Grundschule besuchen, weiß man doch in der Regel gut über deren Schwächen und Stärken in den schulischen Leistungen Bescheid - auch im Vergleich mit anderen Kindern der Klasse. Gelegentlich weiß man auch, oder meint zu wissen, dass die Grundschule A an einem Ort „besser“ ist als die Grundschule B, was immer dieses ",besser“ im Hinblick z. B. auf Schulleitung, Lehrerkollegium, Unterricht, Zusatzangebote, Schülerleistungen, Sozialstatus der Schülereltern, heißen mag. Auch die Bildungsforschung war bis vor gut einem Jahr bei Aussagen zu diesen Punkten in Bezug auf das System Grundschule in der Bundesrepublik bzw. in einzelnen Bundesländern weitgehend auf Vermutungen angewiesen.
\end{abstract}

Inzwischen liegen jedoch mit der in die Internationale Grundschul-Lese-Untersuchung IGLU eingebetteten nationalen Ergänzungsstudie IGLU-E für das Ende der Grundschulzeit, die vierte Jahrgangsstufe, verlässliche nationale Befunde, sowie Befunde für einige Bundesländer von Deutschland über Bildungsvoraussetzungen und Bildungsergebnisse in Mathematik und im Bereich Naturwissenschaften vor (Bos et al. 2003, 2004, Walther et al. 2003, 2004, Prenzel et al. 2003, 2004). Unter Beachtung bestimmter Bedingungen ermöglicht zudem die besondere Konzeption der Studie „rückwirkend“ den Vergleich mit Ergebnissen aus Staaten, die an der internationalen TIMS-Studie von 1995 für Grundschulen beteiligt waren, an der jedoch Deutschland nicht teilgenommen hatte.
Die in den internationalen TIMS-Studien und dem ersten Zyklus der von der OECD initiierten PISAStudie für die beiden Sekundarstufen bzw. die Altersgruppe der 15-Jährigen gewonnenen wenig erfreulichen Ergebnisse in den mathematischen (und naturwissenschaftlichen) Leistungen sorgten bekanntlich in der breiten Öffentlichkeit für Furore. Aber auch die Fachleute, Mathematiker und Mathematikdidaktiker, vertreten durch ihre Fachgesellschaften, zeigten sich in hohem Maße über die Befunde beunruhigt, was seinen Niederschlag in einer Reihe von pointierten Erklärungen der Fachverbände DMV, GDM und MNU fand (Gemeinsame Erklärung der Fachverbände DMV, GDM, MNU vom 19.2. $1997 \mathrm{zu}$ TIMSS II, der DMV vom 6.12.2001 zu PISA, Gemeinsame Erklärung der Fachverbände DMV, GDM, MNU vom 28. 6. 2002 zu PISA-E). Aus diesen Erklärungen spricht unverkennbar der Wille dieser Fachverbände, insbesondere also der von ihnen vertretenen Mathematiker, Verantwortung in einem Teilbereich von Schule, nämlich der mathematischen Bildung in den Sekundarstufen, zu übernehmen. Als Konsequenz: die in internationalen Vergleichsuntersuchungen festgestellten beunruhigenden Leistungsergebnisse deutscher Schüler in Mathematik in den Sekundarstufen haben bei Fachdidaktikern und Mathematikern zu einer Reihe von Maßnahmen geführt, mit dem Ziel, die Qualität von Mathematikunterricht und die mathematischen Kompetenzen von Schülern zu steigern.

Ein deutlich anderes Bild ergab sich bei der Rezeption der Befunde aus IGLU-E. Die Botschaft im ersten Satz der KMK Pressemitteilungen vom 8. 4. 2003 zu den IGLU Ergebnissen dürfte wohl bis heute die 
Richtung der Auseinandersetzung mit diesen Ergebnissen geprägt haben: ${ }^{1}$

Die heute vorliegenden Ergebnisse der internationalen Grundschul-Leseuntersuchung PIRLS/IGLU und der nationalen Erweiterung IGLU-E können für Deutschland insgesamt als erfreulich bezeichnet werden.

In der Tat schnitten unsere Grundschüler in Mathematik im internationalen Vergleich am Ende des vierten Schuljahres deutlich besser ab als bei TIMSS und PISA in der Sekundarstufe I. In der durchaus berechtigten Freude über diese „good news“ gingen offensichtlich in der breiten Öffentlichkeit, zum Teil aber auch in den ,zuständigen“ Fachverbänden die in den weiteren Punkten der KMK Presseerklärung ebenfalls enthaltenen Hinweise auf Defizite bei den Leistungsergebnissen unter.

Damit schien, anders als bei den TIMSS- und PISAErgebnissen, kein unmittelbarer Anlass für ein „Einmischen" insbesondere von Mathematikern an Universitäten, in die Belange des Mathematikunterrichts an Grundschulen oder die mathematische Aus- und Fortbildung von Grundschullehrkräften gegeben zu sein.

Gerade hier liegt nun das Motiv für diesen Beitrag. Mit der Beleuchtung von einigen, durch IGLUE identifizierten, zum Teil defizitären Bereichen in Verbindung mit den mathematischen Leistungsergebnissen erhoffe ich mir von Mathematikern ein verstärktes Interesse an und eine konstruktive „Einmischung" in Fragen des Mathematikunterrichts auch im Bereich Grundschule, einschließlich der Aus - und Fortbildung von Grundschullehrkräften. Diese Hoffnung dürfte im Einklang stehen mit der allgemeinen Forderung von Günther Wildenhain, dem derzeitigen Präsidenten der DMV (Wildenhain 2004): „Die DMV muss sich in allen hochschul- und schulpolitischen Grundfragen positionieren, die unmittelbar die Interessen unserer Wissenschaft tangieren."

Schulische Bildung ist unteilbar. Deshalb muss sich der Blick der an Bildung interessierten Mathematiker auch auf die Grundschule als Teilsystem schulischer Bildung und Erziehung richten, und sie sollten darin - wie in weiterführenden Schulen auch - Verantwortung übernehmen. Einige Ansatzpunkte hierzu seien in Stichworten benannt.

Engagement wäre gefragt zum Beispiel

- auf der lokalen Ebene einer Klasse bzw. Schule durch persönliche Affinitäten (etwa in der Elternrolle, als Elternvertreter);

- bei der Entwicklung von Schulprogrammen (als Experte); $\circ$ ggf. bei der Entwicklung z. B. eines mathematischnaturwissenschaftlichen Exzellenzprogramms;

o bei der Einrichtung von Partnerschaften zwischen Grundschulen und Mathematischen Instituten (analog zur DMV Initiative für Gymnasien);

o bei der Förderung von mathematisch besonders interessierten bzw. talentierten Grundschülern;

- bei Konzepten zu einer auf die beruflichen Anforderungen von Grundschullehrkräften zugeschnittenen mathematischen Aus- und Fortbildung.

In den folgenden Abschnitten beschreibe ich kurz die Testkonzeption von IGLU-E, skizziere die Idee der sog. Kompetenzstufen als inhaltliche Interpretation gewonnener Fähigkeitswerte und berichte einige markante Befunde. Dabei stütze ich mich auf die o. g. Veröffentlichungen der Arbeitsgruppe Mathematik (G. Walther, H. Geiser, R. Langeheine und K. Lobemeier) bei IGLU-E.

\section{Zur Testkonzeption}

Wie oben bereits ausgeführt, fehlte bis zur Durchführung des an IGLU gekoppelten Erweiterungsteils für Mathematik (und den naturwissenschaftlichen Sachunterricht) eine groß angelegte, möglichst viele Bundesländer umfassende Studie mit belastbaren Daten zu mathematischen Kompetenzen von Kindern am Ende der vierten Klasse. Vorliegende Untersuchungen zu mathematischen Kompetenzen von Grundschülern, wie die SCHOLASTIK-Studie (vgl. Weinert \& Helmke 1997) und die Hamburger Lern-Ausgangslagen-Untersuchung LAU (Lehmann \& Peek 1997) waren regional begrenzt und erstreckten sich allenfalls auf ein Bundesland. Die Internationale Grundschul-Lese-Untersuchung IGLU, an der sich auch Deutschland beteiligte, bot nun ein Vehikel, um in dem nationalen Erweiterungsteil IGLU-E im „Huckepackverfahren“ dieses Defizit für Mathematik und den naturwissenschaftlichen Sachunterricht zu beseitigen. An der nationalen IGLU-E Studie beteiligten sich 12 der 16 Bundesländer. Für fünf der beteiligten Bundesländer, nämlich Baden-Württemberg, Bayern, Bremen, Hessen und Nordrhein-Westfalen konnte auf deren Wunsch nach entsprechender Stichprobenplanung auch ein Leistungsvergleich auf Länderebene durchgeführt werden (zu Details vgl. Bos et al. 2004).

Um auch einen nachträglichen internationalen Vergleich mit den Leistungsergebnissen von Staaten zu ermöglichen, die sich, anders als Deutschland, an der internationalen TIMS-Studie für die Grundschule (1995) beteiligt hatten, wurde für den Mathematikleistungstest die Hälfte der insgesamt 58 Testaufga-

\footnotetext{
1 Vgl. auch die unter folgenden Internetadressen angegebenen Hinweise auf Presseveröffentlichungen: http://modelle.bildung. hessen.de/iglu/link/, http://www.stadtschulpflegschaft-bonn.de/index.htm
} 
ben aus dem Aufgabenpool dieser TIMS-Studie übernommen. Die anderen Aufgaben wurden von einer Expertengruppe ausgewählt bzw. neu konstruiert.

Das Rotationsdesign des Tests ermöglichte es, die über sechs Testhefte verteilte große Zahl von 58 Aufgaben von knapp 6000 Viertklässlern (aus 168 Schulen) in der Stichprobe bearbeiten zu lassen.

Die Testkonstruktion von IGLU-E orientiert sich an einer Konzeption von mathematischer Grundbildung, wie sie H. Winter (1995) formuliert hat und prüft Kompetenzen der Schüler bei der Mathematisierung außermathematischer Situationen (Sachrechnen), bei der Bewältigung innermathematischer Aufgaben und beim Lösen von Problemen. Die Aufgaben beziehen sich auf die drei zentralen Inhalts- und Begriffsfelder der Grundschulmathematik, nämlich Arithmetik, Geometrie und Größen/Sachrechnen.

Mit IGLU-E sollten zudem dezidiert mathematische Bildungsergebnisse am Ende der vierten Klasse, also in den meisten Bundesländern am Ende der Grundschulzeit erhoben werden. Deshalb musste geprüft werden, ob der Test tatsächlich auch „nahe“ genug an Kernelementen der Lehrpläne in den beteiligten Bundesländern war. Fachleute, die von diesen Bundesländern benannt wurden und mit den Schnittstellen zwischen Schulaufsicht und Unterrichtspraxis vertraut waren, attestierten dem Test eine hohe curriculare Validität.

Die Bearbeitung von Aufgaben aus den genannten Inhaltsbereichen verlangt von den Schülern, mathematisch tätig zu sein; sie erfordert von den Schülern vielfältige Kompetenzen wie z.B. den Einsatz von Faktenwissen, Begriffen, Fertigkeiten, Standardverfahren, den Umgang mit verschiedenen Repräsentationen (z. B. Text, Bild/Diagramm, Zeichen), das Zusammenfügen mehrerer bekannter rechnerischer oder begrifflicher Lösungsschritte oder Lösungselemente, schöpferisches Denken zur Überwindung von Barrieren bei problemhaften Aufgaben.

Die breite Orientierung an zentralen Inhaltsbereichen und grundlegenden Begriffen und Operationen der Grundschulmathematik beugt einer reduktionistischen Testkonzeption vor, die Schülerleistungen etwa nur durch das Lösen reiner Rechenaufgaben zu den Grundrechenarten und der Reproduktion von Faktenwissen erhebt.

Zwei Aufgabenbeispiele zur Illustration. Es mag erfreuen, wenn knapp $70 \%$ der Viertklässler eine mit dem Standardverfahren der schriftlichen Subtraktion lösbare reine Rechenaufgabe mit vierstelligem Minuend und Subtrahend und vierstelligem Ergebnis, bei deren Berechnung drei Überträge zu berücksichtigen sind, korrekt lösen. ${ }^{2}$ Dass jedoch mehr als ein Viertel der Kinder diese Aufgabe nicht oder nicht richtig bearbeiten zeigt, dass auch solche Aufgaben, die „nur" das Abarbeiten eines am Ende der vierten Klasse verfügbaren Standardalgorithmus erfordern, ihre eigenen Schwierigkeiten bereiten.

Betrachten wir eine im multiple choice-Format gegebene Aufgabe, in die alle drei der oben genannten Begriffsfelder der Grundschulmathematik hineinspielen:

Aus einem dünnen, $20 \mathrm{~cm}$ langen Draht wird ein Rechteck geformt. Das Rechteck ist $4 \mathrm{~cm}$ breit. Wie lang ist es?

Diese Aufgabe erfordert begriffliches Wissen in Form von geometrischen Grundvorstellungen etwa zur Rechtecksfigur, zur Streckenlänge, zum Umfang, sowie das Ausdenken eines Lösungsplans und dessen Ausführung in mindestens zwei Schritten.

Wenig erfreulich ist es, wenn bei dieser Aufgabe nur $17 \%$ der Kinder zum Ziel kommen und fast ein Drittel der Kinder unter den Antwortalternativen den Wert $16 \mathrm{~cm}$ wählen.

\section{Stufen mathematischer Kompetenz im Grundschulalter}

Die ,mathematische Fähigkeit‘ von Grundschülern ist ein Konstrukt, das über die Leistungen der Kinder bei der Bearbeitung von Testaufgaben mit unterschiedlichen Schwierigkeitsgraden erschlossen werden kann. Mit der zur Datenauswertung von IGLUE eingesetzten Item Response-Theorie (IRT), einem probabilistischen Testmodell (vgl. Rost 1996), können die numerischen Werte der Fähigkeit der Schüler und der Itemschwierigkeit auf einer gemeinsamen Skala abgebildet werden. Vor dem Hintergrund der Rahmenkonzeption, die zur Auswahl der Testaufgaben geführt hat, kann nun der erreichte Fähigkeitswert der Person durch entsprechende Kompetenzen beschrieben werden, die zur Lösung der Aufgabe und somit zur Bewältigung der Aufgabenschwierigkeit erforderlich sind. Auf diese Weise wird der Personenfähigkeitswert inhaltlich interpretiert. Dies ist unter curricularen, psychologischen und fachdidaktischen Aspekten besonders bedeutsam.

Die Fähigkeitswerte und damit auch die Schwierigkeitskennwerte der Aufgaben streuen bei IGLU-E im Bereich von 203 bis 828. Die Skala der Fähigkeitswerte konnte durch eine überschaubare Anzahl, nämlich 5 , inhaltlich gut interpretierbarer und gut gegen einander abgrenzbarer Kompetenzstufen gegliedert werden. Damit ist auch eine Klassifizierung der Testaufgaben verbunden. Das inhaltlich Besondere jeder

2 Da diese Aufgabe nicht freigegeben ist, müssen wir uns mit einer Beschreibung der Aufgabe begnügen. 


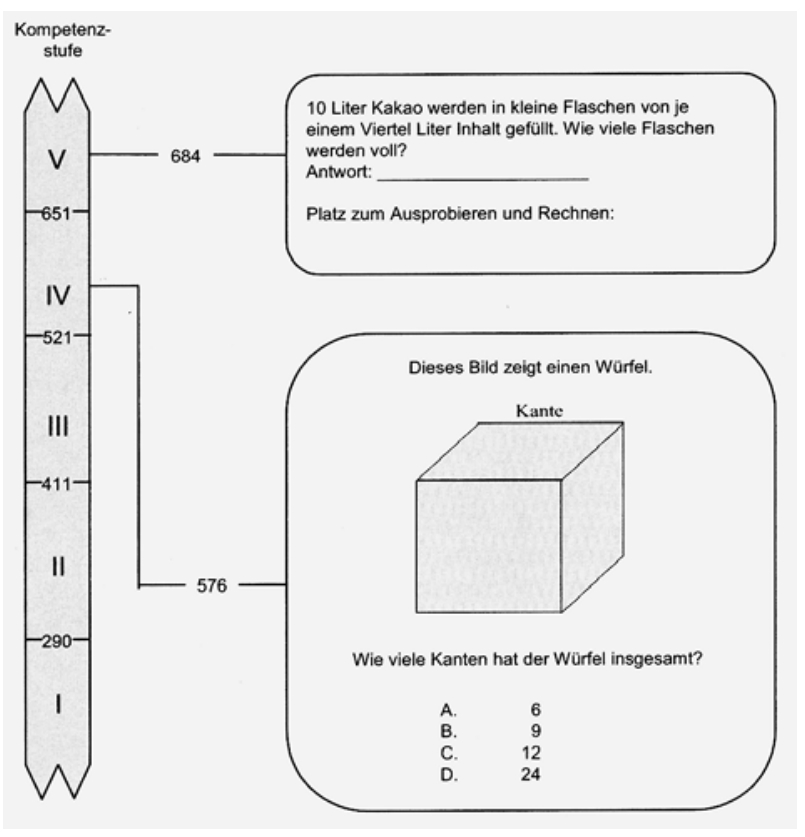

IEA: Progress in International Reading Literacy Study IGLU-Germany

Kompetenzstufe wird durch typische Anforderungen an die mathematische Kompetenz der Schüler bei der Bewältigung der Aufgabenschwierigkeit von charakteristischen Items, sogenannten Markeritems (vgl. die obige Abbildung mit Beispielaufgaben), spezifiziert.

Eine Kurzbeschreibung von Kompetenzen, die auf der jeweiligen Kompetenzstufe (zusätzlich) verfügbar sind, enthalten die folgende Tabelle und der anschließende Abschnitt.

Kompetenzstufen und Skalenbereich

\begin{tabular}{|c|c|c|}
\hline & Kompetenzstufe & $\begin{array}{l}\text { Skalenbereich } \\
\text { der Fähigkeit }\end{array}$ \\
\hline $\mathrm{I}$ & Rudimentäres schulisches Anfangswissen & $<289$ \\
\hline II & $\begin{array}{l}\text { Grundfertigkeiten zum Zehnersystem, zur } \\
\text { ebenen Geometrie und zu Größenverglei- } \\
\text { chen }\end{array}$ & $290-410$ \\
\hline III & $\begin{array}{l}\text { Verfügbarkeit von Grundrechenarten und } \\
\text { Arbeit mit einfachen Modellen }\end{array}$ & $411-520$ \\
\hline IV & $\begin{array}{l}\text { Beherrschung der Grundrechenarten, Be- } \\
\text { wältigung von Aufgaben der räumlichen } \\
\text { Geometrie und begriffliche Modellentwick- } \\
\text { lung }\end{array}$ & $521-650$ \\
\hline $\mathrm{V}$ & $\begin{array}{l}\text { Problemlösen bei Aufgaben mit innerma- } \\
\text { thematischem oder außermathematischem } \\
\text { Kontext }\end{array}$ & $>650$ \\
\hline
\end{tabular}

\section{Ergebnisse}

Nationale Aspekte

Die Verteilung der Viertklässler auf die fünf Kompetenzstufen zeigt die folgende Tabelle:

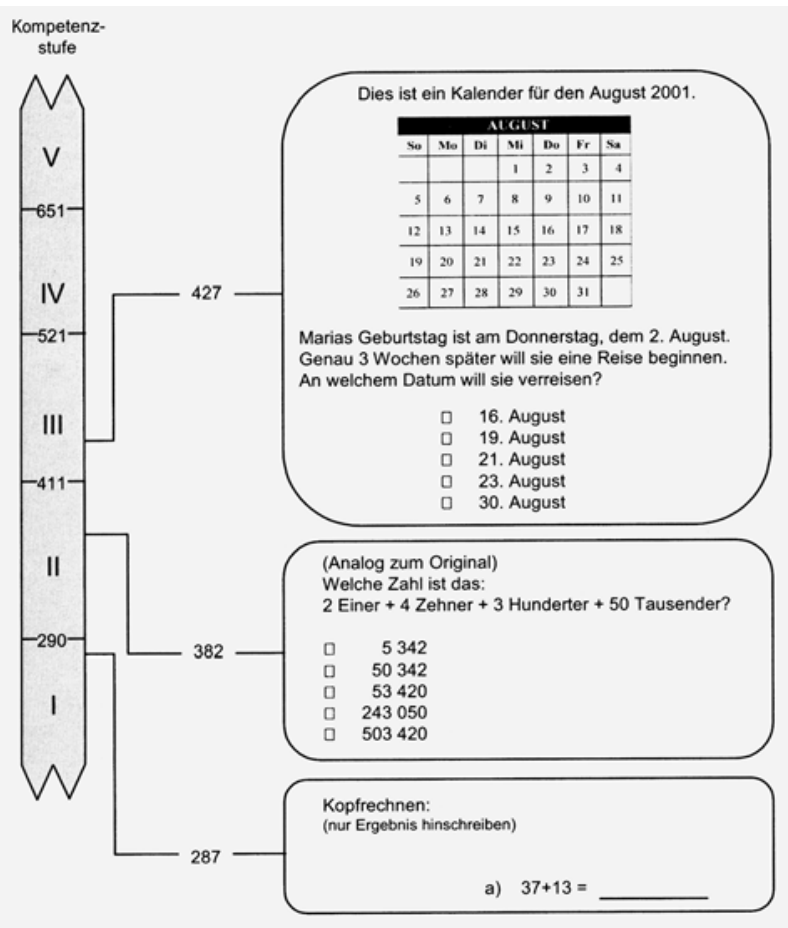

\begin{tabular}{lccccc}
\hline Kompetenzstufe & I & II & III & IV & V \\
Anteil in Prozent & 1,9 & 16,7 & 39,8 & 35,1 & 6,5 \\
\hline
\end{tabular}

IEA: Progress in International Reading Literacy Study IGLU-Germany

Mit Blick auf den Bildungsanspruch der Grundschule, den Anspruch weiterführender Schulen an die erreichten Bildungsergebnisse der Grundschule, und die inhaltliche Beschreibung der Kompetenzstufen, ergibt sich folgendes Bild.

Die gut 40 Prozent der Kinder auf den beiden höchsten Kompetenzstufen genügen ohne Abstriche diesen Ansprüchen. Bei den knapp 40 Prozent der Schüler auf Kompetenzstufe III besteht Nachholbedarf in den drei Inhaltsbereichen Arithmetik, Geometrie, Größen/Sachrechnen, der natürlich besonders groß ist für Kinder, die sich im unteren Teil dieser Kompetenzstufe befinden.

Eine Risikogruppe von fast 20 Prozent der Kinder auf den Kompetenzstufen I und II zeigt erhebliche Defizite in ihren mathematischen Fähigkeiten. Das mathematische Wissen und Können dieser dringend zu fördernden Kinder geht kaum über das zweite Schuljahr hinaus.

Auffällig ist zudem, dass die beiden unteren Kompetenzstufen stärker mit Mädchen als mit Jungen, die beiden oberen Kompetenzstufen dagegen stärker mit Jungen als mit Mädchen besetzt sind.

Da an dieser Stelle keine ausführlichere Erörterung der inhaltlichen Beschreibung der Kompetenzstufen 
erfolgen kann, sei hier nur auf einige für den Grundschulunterricht besonders bedeutsame Befunde verwiesen.

Erst auf Kompetenzstufe IV werden die Grundrechenarten im Zahlenraum bis 10000 mit hinreichender Sicherheit beherrscht. Erst auf Kompetenzstufe IV sind die Kinder bei der Lösung von Aufgaben aus der räumlichen Geometrie erfolgreich. So gelingt es nun etwa, mit Schrägbildern von Würfelbauwerken in unterschiedlichen Anforderungssituationen umzugehen, und z.B. zu entscheiden, welches von mehreren solcher Bauwerke das gedrehte Bild des Originals ist. Kinder auf dieser Stufe können mit begrifflichem Wissen „hinter" das Sichtbare eines Würfels oder von Würfelbauwerken gehen und verdeckte Elemente bei Anzahlbestimmungen berücksichtigen.

Die Schüler auf Kompetenzstufe III können Sachrechenaufgaben mit einfachen Modellierungen lösen bzw. aus gegebenen Modellen Informationen herausholen, verarbeiten und Ergebnisse interpretieren. Auf der nächsten Kompetenzstufe gelingt eine eigenständige begriffliche Modellierung bei Sachrechenaufgaben. Auf Kompetenzstufe V gelingt die Lösung komplexer Sachrechenaufgaben, die zu additiven, multiplikativen und daraus kombinierten mathematischen Modellen führen.

Werfen wir noch einen Blick auf den Anfang dieses Jahres publizierten Vergleich einiger Länder der Bundesrepublik Deutschland (Bos et al. 2004, Walther et al. 2004) bezüglich der Leistungsergebnisse in Mathematik. Der Leistungsmittelwert für Deutschland beträgt auf der internationalen TIMSS-Skala 545 Punkte (Standardfehler $\mathrm{SE}=2,7$ und Standardabweichung $\mathrm{SD}=81$ ) und liegt damit 16 Punkte über dem internationalen Wert für die vierte Jahrgangsstufe der Staaten, die an der TIMS-Grundschulstudie teilgenommen haben. Baden-Württemberg hat im Vergleich der Ländermittelwerte mit 565 Punkten den höchsten, Bremen mit 512 Punkten den niedrigsten Wert erzielt. Der Leistungsmittelwert für BadenWürttemberg liegt signifikant über dem Mittelwert für Deutschland, der für Bremen liegt signifikant darunter. Die Leistungsmittelwerte der anderen Länder (Bayern 547, Hessen 547, Nordrhein-Westfalen 537) unterscheiden sich nicht signifikant vom deutschen Mittelwert.

Länderspezifische Unterschiede ergeben sich u.a. auch bei der Verteilung der Kinder über die fünf Kompetenzstufen und bei den Anteilen der Mädchen und Jungen in den Kompetenzstufen. In Bremen ist im Ländervergleich der Anteil von Viertklässlern in den beiden untersten Kompetenzstufen (Risikogruppe, s. o.) am größten, in Baden-Württemberg am kleinsten. In Bremen gehört etwa jeder dritte Viertklässler dazu, in Baden-Württemberg etwa jeder ach- te. Vergleichbare Anteile weisen die übrigen Bundesländer auf.

In Baden-Württemberg gehört zu den beiden oberen Kompetenzstufen gut die Hälfte, in Bremen gut ein Viertel der Viertklässler. Der Anteil von Kindern in den Kompetenzstufen IV und V in den übrigen Bundesländern entspricht etwa dem nationalen Wert von rund vierzig Prozent.

Interessant ist auch der Vergleich der beiden Länder Baden-Württemberg und Bayern hinsichtlich ihres Abschneidens bei IGLU-E und bei PISA. BadenWürttemberg liegt mit den Leistungsmittelwerten in beiden Studien über den Mittelwerten für Deutschland und kann somit sein vergleichsweise hohes Leistungsniveau am Ende der Grundschule auch in der Sekundarstufe I halten. Bayern liegt am Ende der Grundschule im Bereich des nationalen Mittelwertes; bei PISA hingegen ist Bayern im oberen Mittelbereich anzutreffen, in dem sich auch BadenWürttemberg befindet. Bayern zeigt damit, dass es in der Sekundarstufe I möglich ist, die mathematischen Leistungsergebnisse noch deutlich zu verbessern. Diese Entwicklung ist keineswegs selbstverständlich: der Leistungsmittelwert in Mathematik am Ende der Grundschule liegt für Deutschland deutlich über dem internationalen Mittelwert, bei PISA hingegen fällt Deutschland in die untere Hälfte einer Mittelgruppe von 16 Staaten (Baumert et al. 2001).

\section{Internationale Aspekte}

Wie oben bereits erwähnt, wurde bei IGLU-E als Bezugsgröße für den internationalen Vergleich die TIMS-Grundschulstudie von 1995 herangezogen. Wegen der nicht unerheblichen zeitlichen Distanz zu dieser Studie ist bei der nachträglichen Einordnung der deutschen Testergebnisse Behutsamkeit angebracht (vgl. hierzu Walther et al. 2003).

Die Einordnung des durchschnittlichen Leistungswertes (545 Punkte) deutscher Schüler in Mathematik in die TIMSS-Fähigkeitsskala von 1995 zeigt Deutschland auf einem vergleichbaren Niveau mit Slowenien, Irland, Ungarn, Australien, USA und Kanada. Die durchschnittlichen Leistungswerte in den Staaten dieser Gruppe liegen über dem internationalen Mittelwert von 529 Punkten. Obwohl dieser Abstand zum internationalen Mittelwert für Deutschland deutlich ist, gibt es weitere sieben Staaten, deren Leistungskennwerte wiederum erheblich besser sind als der deutsche Mittelwert. Kurzum: Deutschland gehört mit den Mathematikleistungen seiner Schüler am Ende der Grundschule nicht zur internationalen Spitzengruppe. Der von deutschen Grundschülern erreichte Mittelwert gibt zwar im internationalen Vergleich keine Hinweise auf gravierende Defizite; um aber zur Spitzengruppe aufzuschließen, müsste 
die durchschnittliche Mathematikleistung noch deutlich verbessert werden.

Die Spitzengruppe deutscher Grundschüler kann im internationalen Vergleich durchaus mithalten, wobei allerdings zur Leistungsspitze einiger anderer Staaten noch ein beträchtlicher Abstand besteht. Werfen wir zur Illustration einen Blick auf die fünf Prozent besten Viertklässler in Deutschland. Diese Kinder erreichen mindestens einen Leistungswert von 692 Punkten (Perzentil 95). Die Werte von Perzentil 95 liegen aber z. B. für Japan (726) und Tschechien (711) deutlich höher.

Besonders leistungsfähigen oder an Mathematik besonders interessierten Kindern sollte in der Grundschule ein Angebot zu einer herausfordernden mathematischen Betätigung gemacht werden. Damit könnte die noch vorhandene positive Einstellung der Kinder zur Mathematik weiter ausgebaut, und diese womöglich längerfristig für die Mathematik gewonnen werden.

Der internationale Vergleich kann außerdem dazu beitragen, den Blick für bestimmte nationale Aspekte des Übergangs von der Grundschule zur Sekundarstufe I zu schärfen. Sowohl im Vergleich mit der TIMS-Studie für die Sekundarstufe I als auch im Vergleich mit den PISA Ergebnissen zeigt sich, dass es einer Reihe von Staaten gelingt, ihren Leistungsvorsprung gegenüber Deutschland auf der Basis von TIMSS Grundschule in der Sekundarstufe zu halten oder noch weiter auszubauen. Andere Staaten, wie beispielsweise England und Kanada, die bei TIMSS Grundschule deutlich schlechter abschneiden als Deutschland, erzielen in der Sekundarstufe I etwa bei PISA deutlich bessere Ergebnisse als Deutschland. Somit bedarf es besonderer Anstrengungen der weiterführenden Schulen, das vorhandene Leistungspotential der Grundschüler zu nutzen und die Leistungsentwicklung der Schüler voranzubringen.

Die internationale Einordnung der Leistungsergebnisse für die Bundesländer Baden-Württemberg, Bayern, Bremen, Hessen und Nordrhein-Westfalen kann hier aus Platzgründen nicht mehr berichtet werden; der interessierte Leser sei auf die Literatur
(Walther et al. 2004) verwiesen.

\section{Literatur}

Baumert, J., Klieme, E., Neubrand, M., Prenzel, M., Schiefele, U., Schneider, W., Stanat, P., Tillmann, K.-J. \& Weiß, M. (Hrsg.). 2001. PISA 2000. Basiskompetenzen von Schülerinnen und Schülern im internationalen Vergleich. Opladen: Leske + Buderich.

Bos, W., Lankes, E.-M., Prenzel, M., Schwippert, K., Walther, G. \& Valtin, R. (Hrsg.). (2003). Erste Ergebnisse aus IGLU. Schülerleistungen am Ende der vierten Jahrgangsstufe im internationalen Vergleich. Münster: Waxmann.

Darin: Walther, G., Geiser, H., Langeheine, R. \& Lobemeier, K. (2003). Mathematische Kompetenzen am Ende der vierten Jahrgangsstufe.

Darin: Prenzel, M., Geiser, H., Langeheine, R. \& Lobemeier, K. (2003). Das naturwissenschaftliche Verständnis am Ende der Grundschule.

Bos, W., Lankes, E.-M., Prenzel, M., Schwippert, K., Valtin, R. \& Walther, G. (Hrsg.). (2004). IGLU, Einige Länder der Bundesrepublik Deutschland im nationalen und internationalen Vergleich. Münster: Waxmann.

Darin: Walther, G., Geiser, H., Langeheine, R. \& Lobemeier, K. (2004). Mathematische Kompetenzen am Ende der vierten Jahrgangsstufe in einigen Ländern der Bundesrepublik Deutschland.

Lehmann, R. H. \& Peek, R. (1997). Aspekte der Lernausgangslage von Schülerinnen und Schülern der fünften Klassen an Hamburger Schulen. Bericht über die Untersuchung im September 1996. Hamburg: Behörde für Schule, Jugend und Berufsbildung, Amt für Schule.

Rost, J. (1996). Lehrbuch der Testtheorie, Testkonstruktion. Bern: Huber.

Weinert, F. E. \& Helmke, A. (1997). Entwicklung im Grundschulalter. Weinheim: Psychologie Verlags Union.

Wildenhain, G. (2004). Vorwort des Präsidenten. DMV Mitteilungen, 12-1, 2-3.

Winter, H. (1995). Mathematikunterricht und Allgemeinbildung. Mitteilungen der Gesellschaft für Didaktik der Mathematik, 61, 37-46.

\section{Adresse des Autors}

Prof. Dr. Gerd Walther

Mathematisches Seminar

Universität Kiel

Ludewig-Meyn-Straße 4

24118 Kiel

walther@math.uni-kiel.de 